\title{
tic\&société
}

Vol. $1, n^{\circ} 1 \mid 2007$

De TIS à tic\&société : dix ans après

\section{L'évaluation sociétale des TIC : quelques réflexions sur 25 ans de technology assessment}

Gérard Valenduc

\section{OpenEdition}

Journals

Édition électronique

URL : http://journals.openedition.org/ticetsociete/265

DOI : 10.4000/ticetsociete. 265

Éditeur

Association ARTIC

Référence électronique

Gérard Valenduc, «L'évaluation sociétale des TIC : quelques réflexions sur 25 ans de technology assessment », tic\&société [En ligne], Vol. 1, n¹ | 2007, mis en ligne le 15 février 2008, consulté le 30 avril 2019. URL : http://journals.openedition.org/ticetsociete/265 ; DOI : 10.4000/ticetsociete.265 


\title{
L'évaluation sociétale des TIC : quelques réflexions sur 25 ans de technology assessment
}

\author{
Gérard Valenduc \\ Centre de recherche Travail \& Technologies \\ Rue de l'Arsenal, 5 \\ B-5000 Namur \\ Téléphone +32-81-725122 \\ Fax +32-81-725128 \\ gvalenduc@ftu-namur.org
}

Gérard Valenduc est docteur en informatique, codirecteur de la Fondation Travail-Université et professeur invité aux universités de Namur et Louvainla-Neuve en Belgique. II s'est spécialisé dans la sociologie de la technologie. Ses recherches et publications concernent les mutations du travail liées à la diffusion des TIC, l'exclusion et l'inclusion numériques, les relations entre technologie et société. 


\section{Gérard VALENDUC}

\section{Résumé}

Cet article passe en revue les développements conceptuels et institutionnels du technology assessment (TA) depuis 25 ans, lorsque furent créées les premières institutions de TA en Europe. II accorde une attention particulière à la place des TIC dans cette évolution. La première génération de TA repose sur le concept de choix technologique, développe une évaluation intégrée des différents impacts sociaux et esquisse des scénarios sociétaux. L'anticipation des changements et la démocratisation de la décision politique sont au coeur des activités de TA. Dans les années 90, une seconde génération émerge, basée sur le TA constructif et le TA participatif. Elle est influencée par l'approche constructiviste en sociologie des sciences et vise un façonnage social de la technologie. Alors que les TIC étaient un des sujets préférés de la première génération, leur place au sein de la seconde apparaît plus problématique. L'article discute quelques hypothèses explicatives de cette évolution.

Mots clés : technology assessment ; politique de R\&D ; participation démocratique ; sociologie des sciences et des techniques.

\section{Abstract}

This paper reviews the conceptual and institutional developments of technology assessment (TA) since 25 years, when the first TA institutions were created in Europe, with a particular emphasis on the place of ICT in this evolution. The first generation of TA relies on the concept of technological options, deals with an integrated evaluation of the various social impacts and draws scenarios for society. Anticipation of future changes and democratisation of the political decision making process are at the core of TA activities. In the nineties, a second generation of TA emerges, based on constructive TA and participative TA. This second generation is influenced by the constructivist approach in the sociology of sciences and aims at a social shaping of technology. While ICT were one of the preferred topics of the first TA generation, their place in the second generation appears to be less central. The paper proposes some hypotheses that may explain this change.

Key words: technology assessment; R\&D policy; public participation; sociology of science and technology.

\section{Resumen}

Este artículo revisa los desarrollos conceptuales e institucionales del technology assessment (TA), que han tenido lugar durante 25 años, desde que, en Europa se crearon las primeras instituciones de TA. Se concede 
L'évaluation sociétale des TIC :

quelques réflexions sur 25 ans de technology assessment

una particular atención al papel de las TIC en dicha evolución y se destacan dos generaciones en materia de TA. La primera generación de TA reposa sobre el concepto de opción tecnológica, plantea una evaluación integrada de los distintos impactos sociales y diseña escenarios societales. La anticipación de los cambios y la democratización del proceso de decisión política se sitúan en el núcleo de las actividades TA. A lo largo de los años 90 , emerge una segunda generación, que se fundamenta sobre una TA constructiva y participativa. Esta segunda generación está influenciada por el enfoque constructivista de la sociología de la ciencia y tecnología y apunta a la modelación social de la tecnología. Mientras las TIC eran uno de los temas preferidos de la primera generación, su posición se presenta más problemática en la segunda. El artículo presenta algunas hipótesis que permitan entender este cambio.

Palabras Claves : technology assessment; política de I+D; participación democrática; sociología de la ciencia y de la tecnología 


\section{Gérard VALENDUC}

C'est en 1982 que la France s'est dotée d'un Office parlementaire d'évaluation des choix scientifiques et technologiques (OPECST), dans un objectif de démocratisation de la politique de R\&D et de mise en débat des questions de technologie et société. Ces questions étaient nombreuses et, pour la plupart, toujours d'actualité : l'option nucléaire, les manipulations génétiques, les effets sociétaux de l'informatique et de la télématique, la critique culturelle du savoir scientifique, le rôle des experts dans les débats publics, entre autres. Une réponse institutionnelle se dessinait: la création d'institutions de technology assessment.

Dix ans plus tard, la revue Technologies de l'information et société (TIS) publiait un numéro entièrement consacré à l'évaluation des choix technologiques (vol. $4 n^{\circ} 4,1992$ ), qui s'ouvrait sur un article de Riccardo Petrella intitulé «Le printemps du technology assessment en Europe» (Petrella, 1992). Le technology assessment (TA) connaissait alors de nombreux développements: la mise en place d'institutions de TA dans plusieurs pays européens, une réflexion sur les concepts et les méthodes, un intérêt croissant pour les méthodes participatives de TA, un écho positif dans les programmes européens de recherche, la création d'une communauté scientifique et de réseaux d'experts, la mobilisation d'acteurs sociaux.

Qu'en est-il aujourd'hui du printemps du TA ? Été, automne, hiver ou changement climatique ? De 1982 à 2007, il est intéressant de dresser un bilan de l'évolution contrastée du technology assessment, de ses succès et de ses échecs, de ses illusions et de ses réalisations. Ce bilan concerne le TA en Europe, plus particulièrement en Europe occidentale, car le thème du TA ne figure pas - ou pas encore - parmi les priorités politiques dans les nouveaux pays membres. Évolution des concepts, évolution des institutions : ce sont les deux aspects que traitera cet article. Une attention privilégiée sera accordée à la place des technologies de l'information et de la communication (TIC) dans cette double évolution.

\section{Retour aux sources : la première génération du technology assessment (TA)}

Deux générations successives peuvent être distinguées dans l'évolution institutionnelle et conceptuelle des activités de technology assessment (Valenduc, 2005).

La première génération a assuré la transition entre deux concepts. D'une part, le modèle américain de l'Office of Technology Assessment, créé dès 1972, avait généré un concept de TA apparenté aux études d'impact et aux études de 
L'évaluation sociétale des TIC :

quelques réflexions sur 25 ans de technology assessment

risques (le terme technology assessment se situe dans la lignée de impact assessment et risk assessment, tout comme sa traduction allemande Technikfolgenabschätzung), et marqué par un déterminisme technologique à peine voilé. D'autre part, les premières initiatives européennes, dans les années 1980 , ont développé un concept plus politique, orienté vers les processus de décision et basé sur les notions de choix technologique et de maîtrise de la technologie par la société. L'usage des termes «choix technologiques » ou "options technologiques" dans les traductions latines ou scandinaves de technology assessment est un indice de cette transition.

Une seconde génération d'activités se développe à partir du milieu des années 1990 et s'écarte de plus en plus de la notion de choix technologique, trop empreinte de déterminisme. Prenant en compte certains acquis de la sociologie des sciences et des techniques, notamment le socioconstructivisme ou la notion de social shaping of technology, cette seconde génération se détache progressivement de la relation de causalité entre options technologiques et impacts sociaux pour s'attacher à une conception coévolutionniste des rapports entre technologie et société. De plus, la question du débat public n'est plus seulement considérée comme un moyen ou une méthode, mais comme une fin en soi. Nous y reviendrons plus loin.

Dans une communication présentée en introduction à la première conférence nationale belge de technology assessment, en novembre 1993 (Valenduc et Vendramin, 1994, pp.3-19), nous avons tenté de caractériser la première génération de TA qui était alors arrivée à maturité dans de nombreux pays européens.

Un premier constat est que le terme technology assessment est polysémique. II fait référence à une démarche, une méthode, une appréhension interdisciplinaire et ouverte des développements technologiques, visant à mettre au point des outils à utiliser dans les processus de décision relatifs à la science et à la technologie. Parmi les différentes définitions qui en sont faites, deux ingrédients communs se retrouvent: d'un côté, une forme d'investigation des conséquences au sens large du développement technologique, et de l'autre, un procédé institutionnel de participation démocratique dans le processus décisionnel au sujet des orientations technologiques. De manière plus générale, le technology assessment est " une forme de dialogue entre la R\&D et la société qui vise à développer une meilleure gestion des potentiels technologiques et qui cherche à savoir ce que représente l'optimum technologique en matière de bien-être économique, social mais aussi politique 


\section{Gérard VALENDUC}

et culturel» (Buchs, 1992). C'est auprès des Parlements qu'ont été créées les premières institutions d'évaluation des choix technologiques: aux États-Unis (1972), en France (1982), aux Pays-Bas (1986), au Danemark (1986), en Grande-Bretagne (1987), au Parlement européen (1987), en Allemagne (1989) ${ }^{1}$. Le besoin d'évaluer les choix technologiques a grandi dans un contexte où l'ampleur des développements technologiques et des enjeux des programmes de $R \& D$ rendait nécessaire le développement d'une capacité d'éclaircissement à l'attention des décideurs publics. Plusieurs facteurs ont généré ce besoin d'évaluer les développements technologiques: la peur des conséquences de l'introduction de nouvelles technologies, le besoin de propositions alternatives, la volonté de l'opinion publique d'être prise en compte et enfin, la récession économique qui a largement contribué au développement d'un souci d'évaluation à l'égard des politiques technologiques et industrielles.

Un second constat est que, à partir d'une vocation originelle d'aide à la décision, le technology assessment s'est considérablement élargi et enrichi. Dans sa transcription européenne, il s'est développé non seulement au niveau national, mais également au niveau des institutions européennes et dans certaines entités régionales. Les pratiques et réalisations européennes ont multiplié les finalités et les fonctions du technology assessment, en conjuguant au besoin d'aide à la décision une démarche plus large d'évaluation «sociétale».

À ce stade, cinq caractéristiques permettent de résumer les fonctions principales du technology assessment de «première génération». Elles montrent le chemin parcouru depuis un déterminisme technologique plus ou moins inavoué, hérité de la filiation américaine, jusqu'à la notion de maîtrise sociétale des choix technologiques, typiquement européenne.

- L'aide à la décision. La complexité des innovations technologiques et de leurs enjeux rend difficile la formulation d'un choix ou d'une décision politique sans une compréhension approfondie des implications de ce choix. Il s'agit de concilier les objectifs d'efficience et de démocratisation des choix scientifiques et techniques. Au début des années 90, diverses études mettent en avant le besoin de renforcer l'inscription des travaux de technology assessment dans les processus décisionnels (Smits, 1990 ; Petrella, 1992 ; Smits, Den Hertog et Kuijper, 1993). Elles plaident notamment pour le développement de travaux de nature moins

\footnotetext{
1 Une liste des principales institutions européennes d'évaluation des choix technologiques peut être consultée sur http://www.ftu-namur.org/emerit, avec des liens vers les sites de ces institutions.
} 
L'évaluation sociétale des TIC :

quelques réflexions sur 25 ans de technology assessment

académique, orientés vers les usagers du technology assessment, et qui privilégient un rôle de conseiller plutôt que d'expert.

- L'approche sociétale. Le principe de l'approche sociétale de la technologie consiste à envisager tous les aspects corrélés d'une manière ou d'une autre à un choix technologique ou à un programme de R\&D. II s'agit non seulement d'apprécier ce choix à partir de critères économiques ou techniques, mais aussi d'inclure dans l'évaluation des aspects sociaux, environnementaux, éthiques, etc. L'originalité du technology assessment réside dans l'intégration de ces différentes dimensions.

- L'évaluation prospective. Celle-ci met en oeuvre une capacité d'anticipation des tendances technologiques, de leurs potentialités et de leurs contraintes, à des fins de gestion prévisionnelle des innovations futures et des conditions de diffusion de ces innovations. L'évaluation prospective peut comprendre la veille technologique, la détection des problèmes et des externalités négatives, la construction de scénarios, la contribution au développement des politiques futures, la formulation et le développement d'applications technologiques socialement souhaitables, etc.

- La prise en compte des controverses et des incertitudes. Le technology assessment est confronté à des choix technologiques qui relèvent d'une dynamique complexe. Les décideurs privés et publics se trouvent face à des incertitudes ou pris dans des controverses. Contrairement à une attitude qui prévalait dans les années 80 , les incertitudes et les controverses ne sont plus considérées comme un frein, mais comme une opportunité créatrice pour le technology assessment (Lascoumes, 2001). Cette prise en compte des controverses scientifiques et techniques se concrétise non seulement dans les travaux de recherche, mais également à travers des méthodes et des manifestations qui mettent ensemble les différents acteurs d'une controverse, qui tentent de l'élucider et de mettre en évidence les alternatives éventuelles.

- L'ouverture au débat public. Celle-ci s'effectue en confiant aux pratiques de technology assessment une vocation d'interface entre la recherche et la société. L'ouverture au débat public élargit l'information disponible et la prise en compte de l'opinion publique. Elle contribue également à encourager l'acceptation des technologies par le grand public et à développer une certaine culture technique. Elle concrétise le modèle pragmatique proposé dès 1964 par Jürgen Habermas, dans lequel « les 


\section{Gérard VALENDUC}

recommandations techniques et stratégiques ne peuvent s'appliquer efficacement à la pratique qu'en passant par la médiation politique de l'opinion publique. En effet, le dialogue qui s'établit entre les experts spécialisés et les instances de la décision politique détermine la direction du progrès technique à partir de l'idée qu'on se fait de ses besoins pratiques, en fonction d'une certaine tradition, tout autant qu'il critique et mesure cette idée aux chances que la technique lui donne de voir ses besoins satisfaits ; et ce dialogue doit justement être en prise directe sur les intérêts sociaux et les orientations d'un monde vécu social donné par rapport à certaines valeurs » (Habermas, 1991, p.109). Toutefois, bien qu'elle soit aujourd'hui entrée dans les moeurs, l'association des termes science, technologie et débat ne va pas de soi. Selon une opinion largement répandue, héritée de Max Weber, le débat public est fait d'opinions, d'aspirations, de controverses, d'incertitudes, d'alliances et de rapports de pouvoir - c'est-à-dire d'irrationnel. En revanche, la science et la technologie renvoient bien souvent à d'autres représentations, faites d'objectivité, de rationalité et d'efficacité. L'enjeu du débat public sur les choix technologique est précisément de briser cette image, en montrant tout à la fois que la science et la technologie ont une dimension politique et que la décision politique s'appuie sur une rationalité.

Anticiper, évaluer, débattre : tels sont les principaux objectifs de la première génération du technology assessment. Les nombreuses études de terrain qui ont été menées à travers l'Europe à partir du milieu des années 1980 ont incontestablement fait reculer la conception d'un déterminisme systémique de la technologie. II est apparu de plus en plus clairement que les choix technologiques sont en même temps, et indissociablement, des choix politiques, économiques, sociétaux, juridiques, culturels, pour n'en citer que les principales dimensions.

\section{La seconde génération du TA}

La seconde génération de TA qui est née au milieu des années 1990, se détache du lien de cause à effet entre choix technologiques et implications sociétales. L'accent est mis sur deux dimensions prioritaires. D'une part, le TA constructif: l'évaluation des choix technologiques ne concerne pas seulement l'anticipation des options technologiques envisageables et la mise en scénarios de leurs impacts potentiels, mais aussi l'accompagnement d'un projet technologique tout au long de son cycle de vie. D'autre part, le TA participatif : la participation démocratique n'est plus seulement une méthode de technology assessment, elle devient un objectif en soi. 
L'évaluation sociétale des TIC :

quelques réflexions sur 25 ans de technology assessment

Selon Arie Rip, un des théoriciens du concept de constructive technology assessment (CTA), "l'idée de base est de déplacer le point focal du TA, de l'évaluation des technologies au moment où elles sont déjà constituées vers l'anticipation de leurs effets à un stade précoce de leur développement. Les acteurs au sein du monde de la technologie deviennent alors un groupe cible important, mais le constat que les impacts sont coproduits aux stades d'implémentation et de diffusion - qui est un résultat des études constructivistes - implique que les acteurs du monde de la technologie ne doivent pas être les seuls impliqués. La stratégie à préférer pour le CTA est d'élargir les aspects et les acteurs à prendre en compte. De manière plus générale, le CTA s'achemine vers un processus d'apprentissage sociétal et parfois vers un processus de gestion de la technologie au sein de la société. » (Rip, 1994, p.3). Son collègue Johan Schot surenchérit: " lorsque des utilisateurs, des groupes sociaux ou des citoyens prennent part à un processus de conception, ils sont davantage capables que les concepteurs et ingénieurs de prendre en compte des aspects sociaux à un stade précoce. Les concepteurs anticipent rarement les effets sociaux, ils éprouvent même des difficultés à anticiper à temps les conditions du marché. Ils ne recherchent pas les informations adéquates sur les marchés, et quand ils le font, ils ne se trouvent pas en position d'en faire un usage efficace. Ils réagissent aux signaux du marché et aux effets sociaux seulement quand ils se produisent, ce qui conduit à donner aux problèmes des solutions ad hoc. " (Schot, 1998, p.216)

Dans la pratique, le TA constructif désigne un ensemble de méthodes d'accompagnement et d'évaluation de projets d'amont en aval, avec une implication étroite des acteurs et des parties prenantes (Sundermann, 1999). Rip et Schot formulent trois critères de qualité pour le TA constructif (Schot et Rip, 1997) :

- L'importance de l'anticipation. Tous les aspects sociaux ne peuvent pas être prévus en amont d'un projet de développement technologique, au contraire, ils apparaissent parfois de manière imprévue ou sous la forme d'effets pervers, à tous les stades du projet. Ce constat entraîne deux conséquences. D'une part, l'implication des acteurs concernés doit s'effectuer de manière continue et, d'autre part, le processus de développement technologique doit présenter une certaine flexibilité et pouvoir incorporer des boucles itératives d'essai et d'évaluation.

- La réflexivité. La technologie et ses effets sociaux sont co-produits. Plus précisément, des options technologiques sont liées aux objectifs 


\section{Gérard VALENDUC}

sociaux à prendre en compte et, symétriquement, des choix sociaux sont liés aux trajectoires d'innovation. Les acteurs doivent intégrer à la fois les objets techniques et leurs effets dans leurs réflexions et leurs actions.

- L’apprentissage sociétal. Le développement technologique peut être décrit comme un processus visant à établir de nouveaux couplages entre normes techniques, structures de production, conditions du marché et bases culturelles. Ce processus n'est pas donné, il s'apprend, notamment par le fait que chaque partie concernée doit apprendre à spécifier ses propres critères de conception et à les confronter avec ceux des autres.

Le TA participatif est le complément naturel du TA constructif. Selon Herbert Paschen, fondateur du bureau d'évaluation des choix technologiques auprès du Bundestag, deux facteurs déterminent l'évolution vers des méthodes de plus en plus participatives. D'un côté, la manière dont les institutions de TA s'adaptent pour améliorer leur légitimité et leur efficacité par rapport aux décideurs politiques et aux parlements; de l'autre, le changement de la culture politique qui, à côté de la démocratie représentative, favorise la participation directe des citoyens (Paschen et Vig, 2000). Pour la plupart des institutions européennes de TA, les activités de débat public et de participation directe des citoyens sont devenues indispensables à leur légitimité vis-à-vis de l'opinion publique.

Les méthodes participatives de TA ne sont pas neuves, mais elles sont contraintes à un renouvellement permanent ${ }^{2}$. Ce qui change aujourd'hui, c'est que la participation directe n'est plus une méthode parmi d'autres, mais un objectif en soi. Cet objectif repose sur trois considérations importantes:

- Le rôle de la société civile dans la gouvernance du système européen de recherche. Le plan d'action "Science et société» de la Commission européenne, lancé en 2001, prône une participation accrue de la société civile dans le système de gouvernance de la R\&D en Europe (Jaspers, Banthien et Renner, 2003). Le TA participatif y trouve une nouvelle justification et s'y intègre dans une stratégie politique plus

\footnotetext{
2 Sans entrer dans les détails de ces méthodes, on mentionnera les conférences de consensus, les jurys de citoyens, les groupes focaux, les ateliers des scénarios, les cellules de planning, les forums sociotechniques, les auditions publiques, etc. L'institution de TA du Parlement flamand (VIWTA, Vlaamse instelling voor wetenschappelijk en technisch aspectenonderzoek) a publié un guide pratique des méthodes participatives: Slocum N. (2003), Participatory methods toolkit - a practitioner's manual, VIWTA (Brussel), United Nations University (Brugge), Fondation Roi Baudouin (Bruxelles).
} 
L'évaluation sociétale des TIC :

quelques réflexions sur 25 ans de technology assessment

vaste, mais aussi plus floue - du moins par rapport aux acquis du TA participatif dans certains pays comme le Danemark ou les Pays-Bas.

- La gestion des incertitudes et des risques. Les méthodes participatives ont aussi pour fonction d'évaluer le degré d'acceptabilité des risques technologiques par la société et les modalités de contrôle des risques (Hennen, 1999). Dans des processus de décision où les connaissances scientifiques sont entachées d'incertitudes, la participation du public peut conduire à légitimer des décisions qui ne peuvent pas s'appuyer de manière fiable sur les seuls arguments rationnels.

- Le caractère constructif du TA. L'implication des acteurs concernés tout au long du processus d'innovation nécessite la mise en œuvre de méthodes participatives adéquates, mais plus ciblées que quand il s'agit d'organiser un vaste débat public. Certains auteurs plaident en faveur d'une meilleure intégration du TA constructif et du TA participatif (Berloznik et Van Langenhove, 1998). À nos yeux, cette intégration permettrait d'éviter une double dérive : celle du TA constructif vers une simple technique de management participatif de l'innovation, celle du TA participatif vers une mise en scène de la communication entre le monde de la recherche et le grand public.

Entre la première et la seconde génération du TA, des développements théoriques importants se sont produits dans la sociologie des sciences et des techniques. Un déterminisme technologique adouci mais tenace, souvent sousjacent à la notion de choix technologique, a été battu en brèche par les thèses socioconstructivistes de Michel Callon et Bruno Latour (théorie de l'acteur réseau), ainsi que par d'autres travaux théoriques moins polémiques et moins médiatisés, notamment le modèle de construction sociale de la technologie de Wiebe Bijker et Trevor Pinch (Valenduc, 2005, pp.52-64).

La distinction entre la première et la seconde génération de technology assessment ainsi que l'apport du constructivisme, sont caractérisés par Wiebe Bijker de la manière suivante : "Les premières initiatives de TA reposaient sur l'hypothèse que la connaissance scientifique était capable de formuler des recommandations préventives et pouvait fournir des indications précises dans les décisions relatives au développement technologique. Les scientifiques jouaient alors un rôle privilégié et dominant. Le processus de prise de décision était supposé s'organiser autour de quelques décideurs clairement identifiables (le parlement, le gouvernement, les dirigeants d'entreprises) et il devait pouvoir être amélioré en lui donnant une meilleure rationalité. (...) La conception 


\section{Gérard VALENDUC}

actuelle du TA s'écarte de la précédente sur plusieurs points. Les limites de la connaissance scientifique sont plus clairement prises en compte et, en combinaison avec une reconnaissance de l'importance des sources non scientifiques d'évaluation et de jugement, il y a maintenant un meilleur équilibre entre l'implication des utilisateurs, des producteurs et des décideurs politiques, aux côtés des scientifiques. Le processus de décision politique est maintenant considéré comme fragmenté et diffus - l'existence d'un preneur de décision unique et d'un déroulement rationnel de la procédure de décision sont des illusions. Les décisions ne sont pas rationnelles, mais normatives. " (Bijker, 1995, p.13)

Bijker accorde une grande importance au fait que, dans le processus de construction sociale de la technologie, il n'y a pas, a priori, de groupe social privilégié à impliquer dans un développement technologique, et cela justifie à ses yeux le parti pris méthodologique du TA constructif de s'intéresser à tous les acteurs et toutes les parties prenantes. Le refus d'accorder un rôle a priori privilégié à l'un ou l'autre groupe social entraîne des conséquences méthodologiques, empiriques et politiques: "Sur le plan méthodologique, ce refus de privilégier certains groupes implique un traitement symétrique de tous les groupes sociaux pertinents, à travers les mêmes lunettes conceptuelles et sans hypothèses préalables sur ce que sont leurs intérêts spécifiques, leurs compétences ou leurs positions dans la société. Sur le plan empirique, cela signifie que, pour comprendre un développement technologique concret, nous devons regarder au-delà de ceux qui sont concernés à première vue, les techniciens, les gestionnaires et les gens du marketing. Le processus de construction sociale continue bien après que les artefacts aient quitté l'atelier. Sur le plan politique, (...) affirmer qu'il n'y a pas de groupe préalablement privilégié est une prise de position dans le débat sur la culture technologique. Tous les groupes sociaux pertinents sont d'une importance égale " (Bijker, 1995, p.19). Bijker considère donc que les acquis du constructivisme social modifient fondamentalement les notions de choix technologique et de négociation des choix telles que formulées dans la première génération du TA.

Ce point de vue de Bijker est moins radical que le principe d'agnosticisme par rapport aux positions et stratégies des acteurs, défendu par l'école socioconstructiviste de Callon et Latour (Vinck, 1995, pp.196-200), mais il ne fait pas l'unanimité parmi les protagonistes du TA. Ceux-ci évoluent dans un contexte où les rapports de force entre acteurs apparaissent au premier plan et où l'accès à l'information scientifique et à l'expertise constitue un facteur d'inégalité entre les groupes sociaux concernés. Bien que le point de vue de Bijker puisse s'avérer créatif sur le plan méthodologique, son opportunité politique est fortement dépendante du contexte. 
L'évaluation sociétale des TIC :

quelques réflexions sur 25 ans de technology assessment

Arie Rip met également en évidence d'autres apports originaux du constructivisme. Le point fort des études socioconstructivistes est l'analyse des innovations au niveau micro, alors que le technology assessment envisage souvent les changements technologiques au niveau macro et en termes de tendances structurantes à long terme. L'intérêt du TA constructif pour le niveau méso permet de rapprocher les points de vue et de concilier une analyse de la construction des innovations avec leur positionnement sociopolitique à plus large échelle. Rip souligne que les chercheurs dans le domaine des études sociales sur la technologie peuvent aussi offrir aux acteurs de terrain ce qu'il appelle un "service professionnel »: c'est-à-dire élaborer des concepts pertinents pour l'action, aider les praticiens à mettre un nom sur des constats, comparer les expériences, cadrer des situations particulières en regard de la littérature et de l'état de l'art des recherches, toutes choses qu'un processus participatif seul ne peut pas produire de lui- même (Rip, 1994, p.5).

\section{L'évolution institutionnelle du TA}

Au début des années 1990, l'institutionnalisation du TA était envisagée sous plusieurs formes : auprès des Parlements, auprès d'assemblées consultatives régionales, à travers des missions à long terme confiées à des instituts scientifiques ou à des réseaux d'institutions. La Commission européenne jouait un rôle actif dans la promotion du TA.

Le modèle parlementaire a toujours été dominant, mais il s'est développé selon deux variantes: une variante discursive et une variante instrumentale (Delvenne et Brunet, 2006). Dans le modèle parlementaire discursif, le rôle du TA est d'organiser un débat public dans la société, puis d'analyser ce débat et d'en proposer une synthèse aux parlementaires et aux décideurs (Allemagne, Danemark, Pays-Bas, Suisse). Dans le modèle parlementaire instrumental, le TA sert essentiellement à fournir une expertise indépendante et pluridisciplinaire (Parlement européen, Finlande); certaines institutions (Grande-Bretagne, France) se rattachent au modèle instrumental, mais prennent de temps à autre des initiatives discursives, notamment des conférences de citoyens. Les institutions parlementaires de TA ont constitué le réseau EPTA (European Parliamentary Technology Assessment, www.eptanetwork.org) qui tient, depuis plus de quinze ans, des réunions scientifiques annuelles et qui participe à des projets de recherche européens. 


\section{Gérard VALENDUC}

Au début des années 1990, le modèle parlementaire de TA coexistait avec un modèle de concertation sociale régionale, reposant sur une implication des "forces vives" du développement technologique régional de manière décentralisée (Carré et Valenduc, 1991). La Stichting Technologie Vlaanderen (STV), en Flandre, et l'Académie de TA du Bade-Württemberg en étaient les réalisations phares, mais des initiatives existaient également dans d'autres régions allemandes, belges, espagnoles ou italiennes. Un réseau européen de TA régional s'était constitué avec le soutien de la Commission européenne.

Avec le temps, ce modèle de concertation sociale régionale a disparu. La STV a été absorbée par le Conseil économique et social flamand; son champ d'activité s'est recentré sur le thème innovation et travail - ce qui ne l'empêche pas de continuer un excellent travail, mais en dehors du champ du TA. Privée du soutien du gouvernement régional, l'Académie de TA du Bade-Württemberg a été dissoute en 2003. L'Institut Arbeit und Technik, à Gelsenkirschen, point focal de diverses initiatives de TA régional en Rhénanie Westphalie, a été fermé en 2006. Aucune autre initiative régionale des années 1990 ne survit encore aujourd'hui. L'ancrage du TA régional ${ }^{3}$ auprès des partenaires sociaux (organisations d'employeurs et de travailleurs) ne s'est donc pas avérée fructueuse. Plusieurs hypothèses explicatives peuvent être avancées. Le principe de la concertation sociale qui est efficace dans le domaine du travail et du développement économique, n'est pas facilement transposable au domaine de la recherche et de l'innovation. En outre, les partenaires sociaux traditionnels sont peu ouverts aux formes de participation directe des citoyens dont ils se méfient, car ils sentent leur représentativité menacée. Enfin, ils entretiennent des rapports difficiles avec la recherche interdisciplinaire et la gestion des controverses.

Si la plupart des centres de TA créés à l'initiative des parlements à la fin des années 1980 se sont imposés comme de bonnes pratiques et ont confirmé leur notoriété, peu de créations institutionnelles récentes ont suivi ce modèle, à l'exception de la Flandre en 2002. Le noyau du TA européen est resté assez stable depuis une quinzaine d'années. Les méthodes de travail, notamment les méthodes participatives de débat public, se sont affinées, mais elles restent dans le filiation de celles qui ont été expérimentées par les précurseurs. En

\footnotetext{
${ }^{3}$ Nous fûmes pourtant parmi les partisans de cette formule. De 1992 à 2002, la Région wallonne a confié à la Fondation Travail-Université la mise en œuvre d'un programme régional de sensibilisation au TA, dénommé EMERIT (Expériences de médiation et d'évaluation dans la recherche et l'innovation technologique). II en subsiste toujours un bulletin trimestriel sur l'évaluation des choix technologiques (La Lettre EMERIT, www.ftunamur.org/emerit).
} 
L'évaluation sociétale des TIC :

quelques réflexions sur 25 ans de technology assessment

revanche, les méthodes de communication des institutions de TA ont fortement évolué, essentiellement sous l'influence de la diffusion d'Internet. Internet permet non seulement la diffusion en ligne des résultats des travaux de TA, mais également une multiplication de publications ciblées ainsi que des consultations ou des forums en ligne lors de la préparation de débats publics. Toutefois, ceux-ci se déroulent toujours dans le monde réel et pas dans le monde virtuel : le cyber-TA n'est pas à l'ordre du jour.

Faut-il attribuer ces succès au modèle parlementaire lui-même ? Dans une certaine mesure, oui, puisque les autres modèles n'ont pas fait leurs preuves. Toutefois, il existe une grande variété parmi les institutions de type parlementaire, selon leur degré d'autonomie par rapport au parlement, leur capacité propre de recherche, leurs stratégies de communication. Par exemple, sur le plan du fonctionnement institutionnel, il y a peu de points communs entre l'Office parlementaire français et l'Institut Rathenau aux Pays-Bas. De plus, le succès de l'évaluation des choix technologiques n'est pas seulement tributaire d'une institutionnalisation réussie, mais aussi d'autres facteurs tels une culture politique de débat sociétal sur la recherche et l'innovation, une capacité d'expertise pluridisciplinaire et une implication des acteurs de terrain.

\section{TIC et TA}

Dans la première génération de TA, les technologies de l'information et de la communication (TIC) ont occupé une place importante, en particulier dans la perspective d'une "maîtrise sociale " des choix technologiques. Les TIC permettaient une mise en œuvre du TA à la fois au niveau macro-social, à travers une approche critique du développement de la société de l'information, et au niveau microsocial, dans les processus de négociation des nouvelles technologies dans les entreprises.

II est intéressant de s'arrêter un moment sur le lien entre la première génération de TA et la négociation des nouvelles technologies. C'est lors du "printemps du TA ", pour reprendre l'expression déjà citée de Riccardo Petrella, que se développent en Europe les premières réalisations du dialogue social face aux changements technologiques: conventions collectives sur l'introduction des nouvelles technologies, lois sur l'expression des salariés, organisation d'une expertise technologique au service des syndicats (Carré et Valenduc, 1991). Les organisations syndicales européennes s'impliquent dans la promotion institutionnelle du TA. Des méthodes sont développées pour 


\section{Gérard VALENDUC}

favoriser des démarches de TA dans les relations sociales (Valenduc et Vendramin, 1992).

Pourquoi les TIC offrent-elles une bonne prise à l'objectif de maîtrise sociale des choix technologiques? La réponse se trouve dans la nature même des TIC, car on y rencontre à la fois les effets structurants des systèmes technologiques et le façonnage de ces systèmes par les acteurs sociaux. Les informaticiens en ont une perception intuitive. Ils construisent des systèmes d'information et des applications logicielles dont ils savent que les usages auront un effet structurant sur le fonctionnement des entreprises, l'organisation du travail, les représentations mentales et les compétences des utilisateurs, la qualité de la vie, la communication dans la société. Toutefois, cette construction est ellemême déterminée par une série de spécifications qui ne sont pas de nature technique telles que les politiques publiques, les stratégies des entreprises, les exigences des clients, les préférences des consommateurs, les mécanismes du marché, les contraintes et opportunités du changement organisationnel, les qualifications disponibles ou les trajectoires sociotechniques préexistantes.

Les TIC présentent quelques caractéristiques spécifiques qui les distinguent des autres systèmes techniques. La première réside dans les notions de système et de programme qui sont au cœur de l'informatique. La notion de système fait référence à une technologie structurée, englobante, faite de relations hiérarchisées et susceptible d'étendre ses impacts au-delà de son champ d'application. La notion de programme, en revanche, renvoie à une technologie flexible et modulable selon les projets qu'elle sert. Sommairement, on pourrait dire que l'aspect programme est socialement construit, tandis que l'aspect système peut s'avérer déterministe.

Une deuxième caractéristique des TIC est leur malléabilité, qui repose essentiellement sur la composante logicielle. C'est à travers le logiciel que les objectifs d'un projet informatique se réalisent. Le logiciel est conçu pour atteindre ces objectifs, il incorpore des valeurs et des rapports sociaux particuliers; les différents groupes sociaux impliqués ou affectés par un projet informatique peuvent y inscrire des priorités et des buts différents. C'est cette malléabilité qui permet différentes modalités de façonnage social au cours du cycle de vie d'un projet informatique (Woolgar et Grint, 1991). Toutefois, la malléabilité n'est pas sans limite. Les contraintes d'intégration des logiciels et de performance managériale peuvent introduire d'importantes rigidités dans les systèmes d'information des entreprises. Malléabilité et rigidité sont deux facettes d'une même interaction des TIC avec les organisations.

Troisièmement, les TIC sont des technologies génériques qui se diffusent dans l'ensemble de l'économie et de la société et interagissent avec celles-ci. 
L'évaluation sociétale des TIC :

quelques réflexions sur 25 ans de technology assessment

Les TIC concernent aussi bien le travail que l'organisation économique, le fonctionnement des états, la culture et la communication. Le vocabulaire glisse imperceptiblement des technologies de l'information à la société de l'information ; la dimension sociétale est incontournable.

Enfin, les TIC sont aussi des technologies de transformation du travail, dont l'utilisation poursuit des finalités économiques : rationalisation des procédés de production de biens et de services, réorganisation du travail, contrôle des performances. Les TIC sont mises au service de stratégies socioéconomiques. Dans ce domaine, les rôles des TIC sont à la fois le reflet de ces stratégies et l'instrument par lequel se manifestent les impacts sociaux sur l'emploi, les qualifications et compétences, les conditions de travail.

Ces caractéristiques spécifiques des TIC peuvent expliquer pourquoi elles ont occupé une place importante dans la première génération de TA. Qu'en estil aujourd'hui ? L'expansion d'Internet, qui coïncide chronologiquement avec l'émergence de la seconde génération du TA, aurait-elle modifié la donne?

La séquence "options technologiques - débat - choix politique ou stratégique - aménagement et contrôle des conséquences " ne semble plus correspondre aux modalités actuelles du changement technologique et organisationnel. Celles-ci sont plus proches d'un processus itératif, avec des phases de tâtonnement, d'essai et d'erreur, d'ajustement, d'accélération ou de temporisation, que d'un processus de décision linéaire et rationnel. Ceci ne veut pas dire qu'il n'y a plus de choix technologiques, mais que ces choix résultent davantage d'orientations, de jeux d'influence, d'interactions et d'adaptations successives - ou d'effets de verrouillage ou d'engrenage. De plus, il ne faut pas oublier que, quelle que soit la complexité de ce processus, il subsiste toujours des moments clés de décision: la décision d'investir ou d'engager des dépenses, de prendre une mesure politique. Ces moments deviennent pourtant plus difficiles à identifier et le débat sur les options plus difficile à mener.

La notion de négociation des choix technologiques a donc perdu beaucoup de sa pertinence, du moins dans le domaine des TIC - cette dernière précision est importante, car la négociation des choix technologiques est toujours à l'ordre du jour dans d'autres domaines, comme les décisions en matière d'environnement, de risques majeurs, de biosécurité, etc. La négociation sociale des TIC s'est-elle pour autant éteinte ? Elle s'est plutôt déplacée vers des thèmes qui balisent le contexte social de la diffusion des TIC, par exemple, l'accès à la formation continue ; les formes de travail flexible basées sur les TIC, 


\section{Gérard VALENDUC}

notamment le télétravail ; les aspects de protection de la vie privée et de contrôle individualisé sur le travail dans l'usage d'internet ou d'un intranet.

Josée Van Eijndhoven, qui dirigea longtemps l'institut de TA créé par le parlement hollandais (Institut Rathenau) avant de devenir rectrice de l'Université Erasmus de Rotterdam, exprime une opinion assez critique au sujet de la place des TIC dans le TA de seconde génération. Dans un article intitulé «Vingt ans de TIC et société : de lourds rapports et un débat bien léger » (Van Eijndhoven, 1999), elle s'interroge sur l'écho relativement faible des questions liées aux TIC dans les débats publics sur la science et la technologie aux PaysBas. Elle souligne que, il y a vingt ans déjà, l'agenda du TA dans le domaine des technologies de l'information a été fixé en termes d'exploration et d'inventaire des impacts potentiels de différents scénarios, essentiellement parce que le développement des TIC était considéré comme largement imprévisible et non déterministe. Les discussions entre experts, décideurs politiques et représentants des acteurs sociaux étaient formulées en termes de politique générale, peu propices à une implication des citoyens dans un débat public.

L'émergence du TA constructif a permis de mener des études plus concrètes, par exemple sur les usages des TIC dans le domaine de la santé, sur les usages des cartes à puces dans des applications de la vie quotidienne, ou encore sur les services gouvernementaux en ligne. Ces études ont conclu au rôle important du façonnage de la technologie par ses usages et ont été instructives pour les décideurs politiques ainsi que pour les concepteurs d'objets techniques et de services en ligne, mais on ne peut pas dire qu'elles aient été très populaires. En général, l'acceptation sociale des TIC est bonne dès le moment où le pouvoir politique prend des mesures pour corriger des inégalités d'accès, favoriser la diversité des usages et prévenir les effets pervers. Le seul thème controversé, qui ait trouvé écho dans le débat public, est celui de la vie privée, non seulement en ce qui concerne la protection des données personnelles et les usages intrusifs du courrier électronique, mais aussi en ce a trait aux nouveaux équilibres et nouvelles perméabilités entre sphère professionnelle, sphère publique et sphère privée.

\footnotetext{
${ }^{4}$ L'Institut Rathenau doit son nom à l'auteur principal d'un rapport officiel néerlandais intitulé Maatschappelijke gevolgen van de mikro-elektronica (Conséquences sociétales de la microélectronique), publié en 1979, c'est-à-dire au même moment que le rapport officiel de Simon Nora et Alain Minc sur L'informatisation de la société en France. L'article cité de Josée Van Eijndhoven a été rédigé à l'occasion du vingtième anniversaire du rapport Rathenau.
} 
L'évaluation sociétale des TIC :

quelques réflexions sur 25 ans de technology assessment

Selon Van Eijndhoven, cette situation contraste fortement avec l'intensité des controverses publiques dans d'autres domaines: les biotechnologies dans l'agroalimentaire, les recherches médicales sur les cellules embryonnaires, l'utilisation des informations du code génétique, le clonage, la sécurité de la chaîne alimentaire, les options en matière de prévention et de gestion des déchets, les choix énergétiques, etc. Dans ces domaines, l'agenda de la R\&D est fortement influencé par les débats publics et par les procédures de décision ou de réglementation au niveau politique. Dans le domaine des TIC, en revanche, l'influence de la société sur la direction du changement technologique prend d'autres formes, plus diversifiées, plus ciblées et plus spécifiques à des contextes particuliers.

Quant à l'expansion d'Internet, au cours des dernières années, elle ne semble pas avoir eu une influence importante sur l'évolution des concepts et des méthodes de TA. Tout au plus a-t-elle contribué à diversifier les thèmes d'évaluation des choix technologiques, qui s'étendent désormais à tout ce qui se désigne par des anglicismes en «e-»: e-gouvernement, e-santé, eapprentissage, e-commerce, etc. En revanche, l'usage d'internet a amené des transformations radicales en matière de communication dans le domaine du TA. Toutes les institutions ont aujourd'hui des portails web, des sites documentaires très riches, des publications électroniques, des bulletins en ligne, des productions multimédia. Toutefois, comme on peut le constater à la visite de ces sites, la plupart des institutions «labellisées » TA restent prudentes vis-à-vis des forums, enquêtes en ligne ou autres usages d'internet réputés interactifs.

\section{Développement durable et principe de précaution}

Pour clôturer cet aperçu de l'évolution des concepts et des institutions de TA, il est nécessaire de mentionner deux éléments clés qui n'entraient pas en jeu il y a vingt-cinq ans: la notion de développement durable et le principe de précaution. Ces deux éléments ne sont pas de première importance pour la plupart des questions de TA liées au TIC, mais ils sont essentiels dans les sujets qui sont aujourd'hui en haut de l'agenda dans les institutions de TA et qui relèvent des biotechnologies, de la génétique, de la sécurité alimentaire, du changement climatique, de l'énergie, de la mobilité, etc.

En quoi le développement durable et le principe de précaution modifient-ils le technology assessment ? En quelques mots, le concept de développement durable modifie l'horizon des études prospectives et des scénarios. Le principe 


\section{Gérard VALENDUC}

même de la prospective doit être reformulé. II ne s'agit plus tellement d'extrapoler à long terme les tendances ou options actuelles, mais bien de se fixer un objectif à atteindre dans un horizon temporel précis, puis d'étudier par récurrence quelles sont les différentes voies possibles pour atteindre cet objectif et quel est l'échéancier des mesures à envisager (back-casting au lieu de forecasting). Quant au principe de précaution, il a mis en avant la notion d'incertitude scientifique. L'incertitude scientifique provient du fait que les connaissances existantes apparaissent, à un moment donné, incomplètes ou insuffisantes pour estimer correctement la probabilité d'un risque ou les conséquences de ce risque ; il devient alors difficile de légitimer ou d'étayer des décisions politiques à partir d'avis scientifiques (POST, 2004). Se référer au principe de précaution, c'est accepter que des critères relatifs à l'avenir de la vie en société puissent modifier de manière significative la logique de l'innovation technologique (Salomon, 2005). C'est aussi introduire de nouvelles modalités dans le développement technologique comme celles de la réversibilité de certains choix, la réévaluation continue de l'état des connaissances par rapport à l'état des controverses ou, enfin, la primauté du long terme.

Ces enjeux sont sans doute ceux des vingt-cinq prochaines années de technology assessment, mais le propos de cet article était plus modestement de développer une réflexion sur les vingt-cinq années passées. 
L'évaluation sociétale des TIC :

quelques réflexions sur 25 ans de technology assessment

\section{Références bibliographiques}

BERLOZNIK R. et L. VAN LANGENHOVE, 1998, «Integration of technology assessment in R\&D management practices ", Technological forecasting and social change, vol. 58, n¹/2, pp.23-33.

BIJKER W., 1995, "Democratisering van de technologische cultuur », dans Inaugurale rede van de leerstoel Techniek en Samenleving, le 24 mars, Rijksuniversiteit Limburg te Maastricht.

BUCHS T., 1992, Technology assessment: expériences occidentales et défis actuels, Bern, Rapport au Conseil suisse de la science n`131.

CARRÉ D. et G. VALENDUC, 1991, Choix technologiques et concertation sociale, Paris, Economica.

DELVENNE P. et S. BRUNET, 2006, « Le technology assessment en question: une analyse comparative ", Courrier hebdomadaire du CRISP, $\mathrm{n}^{\circ} 1909$ 1910.

HABERMAS J., 1991, La technique et la science comme idéologie, Paris, réédition Gallimard/Idées.

HENNEN L., 1999, "Participatory technology assessment: a response to technical modernity ?", Science and Public Policy, vol. 26, n5, pp.303-312.

JASPERS M., H. BANTHIEN et A. RENNER, 2003, Governance of the European Research Area: the role of civil society, IFOK report for the European programme Science \& society, DG Research.

LASCOUMES P., 2001, "La productivité sociale des controverses", dans Actes du séminaire Penser les sciences et les techniques dans les sociétés contemporaines, Centre Alexandre Koyré d'histoire des sciences et des techniques, Paris, <http://histsciences.univ-paris1.fr/penserlessciences/>

PASCHEN H. et N.J. VIG, 2000, «Parliaments and technology: the development of TA in Europe ", Summary review in TA-Datenbank Nachrichten, ITAS Karlsruhe, vol. 9 n¹, pp.96-102. 


\section{Gérard VALENDUC}

PETRELLA R., 1992, «Le printemps du technology assessment en Europe : faits et questionnements », Technologies de I'Information et Société, vol. 4, $\mathrm{n}^{\circ} 4$, pp. $425-434$.

POST (Pariamentary Office of Science and Technology), 2004, Handling uncertainty in scientific advice, Postnote 220, London, Pariamentary Office of Science and Technology.

RIP A. 1994, "Science \& technology studies and constructive technology assessment », EASST Review, vol. 13, n³, pp.23-36.

SALOMON J.J., 2005, «Précaution et démocratie », Futuribles, n³11, pp.5-23.

SCHOT J. et A. RIP, 1997, "The past and future of constructive technology assessment », Technological forecasting and social change, vol. 54, n²/3, pp.251-268.

SCHOT J., 1998, "Constructive technology assessment comes of age: the birth of a new politics of technology », dans A. JAMISON (dir.), Technology policy meets the public, PESTO Papers II, Aalborg University, Danmark, pp.207-232.

SMITS R., 1990, State of the art of technology assessment in Europe, Report to the Second European Congress of Technology Assessement, du 14 au 16 novembre, Milan, Italie.

SMITS R., P. DEN HERTOG et J. KUIJPER, 1993, Towards a strong and effective European Technology Assessment Infrastructure, TNO Report for the European Commission, March, Apeldoorn, Pays-Bas.

SUNDERMANN K., 1999, “Constructive technology assessment », dans S. BÖCHLER, G. SIMONIS et K. SUNDERMANN, Handbuch für Technikfolgenabschätzung, Berlin, Sigma Verlag, pp.119-128.

VALENDUC $G$. et P. VENDRAMIN, 1992, «La prospective sociale: une méthode d'évaluation des choix technologiques", Technologies de l'information et société, vol. 4, $\mathrm{n}^{\circ} 4$, pp.457-472.

VALENDUC G. et P. VENDRAMIN, 1994, « Le technology assessment: évolution conceptuelle et institutionnelle en Europe ", dans Technologie et société - Actes de la première conférence belge de T.A., Politique scientifique fédérale, Bruxelles, vol. I, pp.3-19.

VALENDUC G., 2005, La technologie, un jeu de société - Au-delà du déterminisme technologique et du constructivisme social, Louvain-la-Neuve, Academia Bruylant. 
L'évaluation sociétale des TIC :

quelques réflexions sur 25 ans de technology assessment

VAN EIJNDHOVEN J., 1999, «Twintig jaar ICT en samenleving: zware rapporten en een licht debat ", dans Van micro-elektronica tot mega-ICT, Jaarverslag 1999 Rathenau Instituut, Den Haag, pp.13-23.

VINCK D., 1995, Sociologie des sciences, Paris, Armand Colin.

WOOLGAR S. et K. GRINT, 1991, « Computers and the transformation of social analysis », Science, technology and human values, vol. 16, n³, pp.368-378.

\section{Pour citer cet article :}

Valenduc G., «L'évaluation sociétale des TIC : quelques réflexions sur 25 ans de technology assessment», Revue tic\&société [En ligne], Volume 1, n9, 2ème semestre 2007, Numéros, mis en ligne le : 15/02/2008, URL : <http://revues.mshparisnord.org/lodel/ticsociete/index.php?id=265>. 